International Journal of Pure and Applied Mathematics

Volume 83 No. 1 2013, 213-219

ISSN: 1311-8080 (printed version); ISSN: 1314-3395 (on-line version)

url: http://www.ijpam.eu

doi: http://dx.doi.org/10.12732/ijpam.v83i1.14

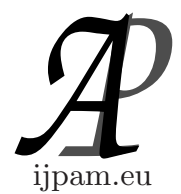

\title{
GENERALIZED HYPERBOLIC METRICS AND DISTORTION OF QUASICONFORMAL MAPPINGS
}

\author{
Mingfeng Sun \\ Department of Mathematics \\ Shaoxing University \\ Shaoxing, Zhejiang, 312000, P.R. CHINA
}

\begin{abstract}
In this paper, we investigate the distortion of generalized hyperbolic metrics under the class $I d_{K}(\partial D)$. We also obtain improved distortion theorems whenever generalized hyperbolic metrics are defined in convex domains.
\end{abstract}

AMS Subject Classification: 30C62, 30C65

Key Words: generalized hyperbolic metric, quasiconformal mappings, distortion, convex domain

\section{Introduction}

There is a classical result of Teichmüller's concerning the distortion of normalized quasiconformal mappings.

Theorem 1. ([1]) Let $h(z, w)$ denote the hyperbolic metric of constant curvature -4 in the three punctured sphere $\mathbb{C} \backslash\{0,1\}$. If $f$ is a $K$-quasiconformal mapping of the Riemann sphere fixing 0,1 and $\infty$, then for any $z \in \mathbb{C} \backslash\{0,1\}$, $h(z, f(z)) \leq \log K$.

Received: January 8, 2013

(c) 2013 Academic Publications, Ltd. url: www.acadpubl.eu 
Let $D$ be a proper subdomain of $\mathbb{R}^{n}$, and let

$I d_{K}(\partial D)=\left\{f: \mathbb{R}^{n} \rightarrow \mathbb{R}^{n}\right.$ is K-quasiconformal $\left.: f(x)=x, \forall x \in \mathbb{R}^{n} \backslash D\right\}$.

It is well known that the class $I d_{K}(\partial D)$ has played an important role both in quasiconformal mapping theory and Teichmüller theory with the case $n=$ 2. There are some generalized hyperbolic metrics which have been studied recently. In this paper, we investigate the distortion of generalized hyperbolic metrics under the class $I d_{K}(\partial D)$. We also obtain improved distortion theorems whenever generalized hyperbolic metrics are defined in convex domains.

\section{Preliminary Results}

Given $E, F \subset \mathbb{R}^{n}$ we denote by $\Delta(E, F)$ the family of all curves that join the sets $E, F$ in $\mathbb{R}^{n}$ and denote by $M(\Delta(E, F))$ its modulus. For a ring domain $R\left(C_{0}, C_{1}\right)$ with complementary components $C_{0}$ and $C_{1}$, we define the modulus of $R\left(C_{0}, C_{1}\right)$ by

$$
\bmod R\left(C_{0}, C_{1}\right)=\left(\frac{\omega_{n-1}}{M\left(\Delta\left(C_{0}, C_{1}\right)\right)}\right)^{1 /(n-1)},
$$

where $\omega_{n-1}$ is the surface area of the unit sphere in $\mathbb{R}^{n}$.

The Teichmüller ring domain $R_{T, n}(t), t>0$ is the doubly connected domains with complementary components $\left[-e_{1}, 0\right]$ and $\left[t e_{1}, \infty\right]$. For the capacity of the Teichmüller ring domain we write $\tau_{n}(t)=\operatorname{cap} R_{T, n}(t)=M\left(\Delta\left(\left[-e_{1}, 0\right],\left[t e_{1}, \infty\right]\right)\right)$.

Lemma 2. ([2]) Let $R=R\left(C_{0}, C_{1}\right)$ be a ring in $\overline{\mathbb{R}}^{n}$, and let $a, b \in C_{0}$, $c, \infty \in C_{1}$ be distinct points. Then

$$
\operatorname{cap} R \geq \operatorname{cap}_{T, n}(t), t=\frac{|a-c|}{|a-b|} .
$$

Equality holds for the Teichmüller ring $R_{T, n}(t)$, with $a=0, b=-e_{1}$, and $c=t e_{1}, t>0$.

Let $x, y \in D \subset \mathbb{R}^{n}$. We denote by $d(x, \partial D)$ the distance between $x$ and the boundary $\partial D$. Let

$$
j_{D}^{\prime}(x, y)=\log \left(1+\frac{|x-y|}{d(x, \partial D)}\right)\left(1+\frac{|x-y|}{d(y, \partial D)}\right) .
$$

The distance ratio metric $j_{D}^{\prime}$ is well known in geometry function theory. It is introduced by Gehring and Osgood in [3]. 
The cross ratio of $z_{1}, z_{2}, z_{3}, z_{4}$ is defined as

$$
\left|z_{1}, z_{2}, z_{3}, z_{4}\right|=\frac{\left|z_{1}-z_{3}\right|\left|z_{2}-z_{4}\right|}{\left|z_{1}-z_{2}\right|\left|z_{3}-z_{4}\right|} .
$$

Apollonian metric is defined as

$$
\alpha_{D}(x, y)=\sup _{a, b \in \partial D} \log (|a, x, y, b|) .
$$

Apollonian metric was first studied by Beardon[4].

Seittentanta metric[5] is defined as

$$
\delta_{D}(x, y)=\sup _{a, b \in \partial D} \log (1+|x, a, y, b|) .
$$

Denote

$$
\delta_{D}^{p}(x, y)=\sup _{a, b \in \partial D} \log \left(1+\left(|x, a, y, b|^{p}+|x, b, y, a|^{p}\right)^{1 / p}\right),
$$

and

$$
j_{D}^{p}(x, y)=\sup _{a \in \partial D} \log \left(1+\left(\frac{|x-y|^{p}}{|x-a|^{p}}+\frac{|x-y|^{p}}{|y-a|^{p}}\right)^{1 / p}\right) .
$$

$j_{D}^{p}$ and $\delta_{D}^{p}$ were studied by Hästö in [6]. Seittenranta has proved the following relation between $\alpha_{D}$ and $\delta_{D}$.

Lemma 3. ([5]) Let $D \subset \mathbb{R}^{n}$ be an open set and Card $\partial D \geq 2$. Then $\alpha_{D} \leq \delta_{D} \leq \alpha_{D}+\log 3$.

In paper [7],the author has proved the following relation among $\alpha_{D}, j_{D}^{\prime}$ and $\delta_{D}^{p}$.

Lemma 4. ([7]) Let $D \subset \mathbb{R}^{n}$ be an open set. Then $j_{D}^{\prime}-\log 9 \leq \alpha_{D} \leq j_{D}^{\prime}$.

Lemma 5. ([7]) Let $D \subset \mathbb{R}^{n}$ be an open set and Card $\partial D \geq 2$. Then $\alpha_{D} \leq \delta_{D}^{p} \leq \alpha_{D}+\log \left(1+2^{\frac{1}{p}+1}\right)$.

\section{The Main Results and Proofs}

In this section we denote by $c(n, K)$ a constant depending only on $n$ and $K$.

Theorem 6. Let $D \subset \mathbb{R}^{n}$ be a domain and $f \in I_{K}(\partial D)$. Then for all $x \in D$

$$
j_{D}^{p}(x, f(x)) \leq 2^{\frac{1}{p}} \log (2+c(n, K)) .
$$


Proof. Let $y=f(x)$. Since $f^{-1} \in I d_{K}(\partial D)$, we may assume $d(x, \partial D) \leq$ $d(y, \partial D)$. Fix $z \in D$ such that $d(x, \partial D)=|x-z|$. For $t>0$, write $F_{t}=$ $\{z+u(z-x): u \geq t\}$. Let $\Gamma_{t}=\Delta\left([x, z], F_{t}\right)$ be the family of all curves in $\mathbb{R}^{n}$ joining $[x, z]$ to $F_{t}$. $\Gamma_{t}^{\prime}=f\left(\Gamma_{t}\right)=\Delta\left(f([x, z]), F_{t}\right)$. From lemma 2 , it follows that

$$
\tau_{n}\left(\frac{t|x-z|}{|y-z|}\right) \leq M\left(\Gamma_{t}^{\prime}\right) \leq K M\left(\Gamma_{t}\right)=K \tau_{n}(t) .
$$

Setting $t=1$, we have

$$
\frac{|y-z|}{|x-z|} \leq \frac{1}{\tau_{n}^{-1}\left(K \tau_{n}(1)\right)} .
$$

Setting $t=|y-z| /|x-z|$, we have

$$
\frac{|y-z|}{|x-z|} \leq \tau_{n}^{-1}\left(\frac{\tau_{n}(1)}{K}\right) .
$$

Hence it follows that

$$
\frac{|y-z|}{|x-z|} \leq \min \left\{\frac{1}{\tau_{n}^{-1}\left(K \tau_{n}(1)\right)}, \tau_{n}^{-1}\left(\frac{\tau_{n}(1)}{K}\right)\right\}=c(n, K),
$$

and

$$
\frac{|x-y|}{d(x, \partial D)}=\frac{|x-y|}{|x-z|} \leq \frac{|x-z|+|y-z|}{|x-z|} \leq 1+c(n, K) .
$$

For any $x>0$ and $p>0$, it is easy to see that $\log \left(1+2^{\frac{1}{p}} x\right) \leq 2^{\frac{1}{p}} \log (1+x)$. Hence

$$
\begin{aligned}
j_{D}^{p}(x, y) & =\sup _{a \in \partial D} \log \left(1+\left(\frac{|x-y|^{\mathrm{p}}}{|x-a|^{\mathrm{p}}}+\frac{|x-y|^{\mathrm{p}}}{|y-a|^{\mathrm{p}}}\right)^{1 / p}\right) \\
& \leq 2^{\frac{1}{\mathrm{p}}} \log \left(1+\frac{|x-y|}{\min \{d(x, \partial D), d(y, \partial D)\}}\right) \\
& =2^{\frac{1}{\mathrm{p}}} \log \left(1+\frac{|x-y|}{d(x, \partial D)}\right) \\
& \leq 2^{\frac{1}{\mathrm{p}}} \log (2+c(n, K)) .
\end{aligned}
$$

Similarly, we can prove the following

Theorem 7. Let $D \subset \mathbb{R}^{n}$ be a domain and $f \in \operatorname{Id}_{K}(\partial D)$. Then for all $x \in D$

$$
j_{D}^{\prime}(x, f(x)) \leq 2 \log (2+c(n, K)) .
$$

By Theorem 7 and Lemma 4, we have the following 
Corollary 8. Let $D \subset \mathbb{R}^{n}$ be a domain and $f \in I d_{K}(\partial D)$. Then for all $x \in D$

$$
\alpha_{D}(x, f(x)) \leq 2 \log (2+c(n, K)) .
$$

By lemma 3 and corollary 8 , we have

Corollary 9. Let $D \subset \mathbb{R}^{n}$ be a domain and $f \in \operatorname{Id}_{K}(\partial D)$. Then for all $x \in D$

$$
\delta_{D}(x, f(x)) \leq 2 \log (2+c(n, K))+\log 3 .
$$

By lemma 5 and corollary 8 , we have

Corollary 10. Let $D \subset \mathbb{R}^{n}$ be a domain and $f \in I_{K}(\partial D)$. Then for all $x \in D$

$$
\delta_{D}^{p}(x, f(x)) \leq 2 \log (2+c(n, K))+\log \left(1+2^{\frac{1}{p}+1}\right) .
$$

In what follows, we will obtain improved distortion theorems of generalized hyperbolic metrics for convex domains.

Theorem 11. Let $D \subset \mathbb{R}^{n}$ be a convex domain and $f \in \operatorname{Id}_{K}(\partial D)$. Then for all $x \in D$

$$
j_{D}^{p}(x, f(x)) \leq 2^{\frac{1}{p}} \log \left(1+\sqrt{c(n, K)^{2}-1}\right) .
$$

Proof. Let $y=f(x)$. Since $f^{-1} \in I d_{K}(\partial D)$, we may assume $d(x, \partial D) \leq$ $d(y, \partial D)$. Fix $z \in D$ such that $d(x, \partial D)=|x-z|$. For $t>0$, write $F_{t}=$ $\{z+u(z-x): u \geq t\}$. Let $\Gamma_{t}=\Delta\left([x, z], F_{t}\right)$ be the family of all curves in $\mathbb{R}^{n}$ joining $[x, z]$ to $F_{t}$. $\Gamma_{t}^{\prime}=f\left(\Gamma_{t}\right)=\Delta\left(f([x, z]), F_{t}\right)$. From lemma 2 , it follows that

$$
\tau_{n}\left(\frac{t|x-z|}{|y-z|}\right) \leq M\left(\Gamma_{t}^{\prime}\right) \leq K M\left(\Gamma_{t}\right)=K \tau_{n}(t) .
$$

Setting $t=1$, we have

$$
\frac{|y-z|}{|x-z|} \leq \frac{1}{\tau_{n}^{-1}\left(K \tau_{n}(1)\right)} .
$$

Setting $t=|y-z| /|x-z|$, we have

$$
\frac{|y-z|}{|x-z|} \leq \tau_{n}^{-1}\left(\frac{\tau_{n}(1)}{K}\right) .
$$

Hence it follows that

$$
\frac{|y-z|}{|x-z|} \leq \min \left\{\frac{1}{\tau_{n}^{-1}\left(K \tau_{n}(1)\right)}, \tau_{n}^{-1}\left(\frac{\tau_{n}(1)}{K}\right)\right\}=c(n, K) .
$$


Since $D$ is convex, $|y-z|^{2} \geq|x-y|^{2}+|x-z|^{2}$. Hence

$$
\frac{|x-y|}{d(x, \partial D)}=\frac{|x-y|}{|x-z|} \leq \sqrt{\left(\frac{|y-z|}{|x-z|}\right)^{2}-1} .
$$

For any $x>0$ and $p>0$, it is easy to see that $\log \left(1+2^{\frac{1}{p}} x\right) \leq 2^{\frac{1}{p}} \log (1+x)$. Hence

$$
\begin{aligned}
j_{D}^{p}(x, y) & =\sup _{a \in \partial D} \log \left(1+\left(\frac{|x-y|^{\mathrm{p}}}{|x-a|^{\mathrm{p}}}+\frac{|x-y|^{\mathrm{p}}}{|y-a|^{\mathrm{p}}}\right)^{1 / p}\right) \\
& \leq 2^{\frac{1}{\mathrm{p}}} \log \left(1+\frac{|x-y|}{\min \{d(x, \partial D), d(y, \partial D)\}}\right) \\
& =2^{\frac{1}{\mathrm{p}}} \log \left(1+\frac{|x-y|}{d(x, \partial D)}\right) \\
& \leq 2^{\frac{1}{\mathrm{p}}} \log \left(1+\sqrt{c(n, K)^{2}-1}\right) .
\end{aligned}
$$

Similarly, we can prove the following

Theorem 12. Let $D \subset \mathbb{R}^{n}$ be a convex domain and $f \in \operatorname{Id}_{K}(\partial D)$. Then for all $x \in D$

$$
j_{D}^{\prime}(x, f(x)) \leq 2 \log \left(1+\sqrt{c(n, K)^{2}-1}\right) .
$$

By Theorem 12 and Lemma 4, we have the following

Corollary 13. Let $D \subset \mathbb{R}^{n}$ be a convex domain and $f \in \operatorname{Id}_{K}(\partial D)$. Then for all $x \in D$

$$
\alpha_{D}(x, f(x)) \leq 2 \log \left(1+\sqrt{c(n, K)^{2}-1}\right) .
$$

By lemma 3 and corollary 13, we have

Corollary 14. Let $D \subset \mathbb{R}^{n}$ be a convex domain and $f \in I_{K}(\partial D)$. Then for all $x \in D$

$$
\delta_{D}(x, f(x)) \leq 2 \log \left(1+\sqrt{c(n, K)^{2}-1}\right)+\log 3 .
$$

By lemma 5 and corollary 13, we have

Corollary 15. Let $D \subset \mathbb{R}^{n}$ be a convex domain and $f \in \operatorname{Id}_{K}(\partial D)$. Then for all $x \in D$

$$
\delta_{D}^{p}(x, f(x)) \leq 2 \log \left(1+\sqrt{c(n, K)^{2}-1}\right)+\log \left(1+2^{\frac{1}{p}+1}\right) .
$$




\section{Acknowledgements}

The research was partially supported by Shaoxing University (No. 20105023)

\section{References}

[1] O. Teichmüller, Ein verschiebungssatz der quasikonformen Abbildung, Deutsche Math., 7 (1944), 336-343.

[2] G.D. Anderson, M.K. Vamanamurthy, M. Vuorinen, Conformal Invariants, Inequalities and Quasiconformal Maps, J. Wiley (1997).

[3] F.W. Gehring, B.G. Osgood, Uniform domains and the quasihyperbolic metrics, J. Anal Math., 36 (1979), 50-74.

[4] A.F. Beardon, The Apollonian metric of a domain in $\overline{R^{n}}$, Quasiconformal mappings and analysis, Springer-Verlag (1998), 91-108.

[5] P. Seittenranta, Möbius invariant metrics, Math Proc Cambridge Philos Soc, 125 (1999), 511-533.

[6] P.A. Hästö, A new weighed metric: the relative metric I, J. Math. Anal. App., 274, No. 1 (2002), 35-58.

[7] Mingfeng Sun, Gromov hyperbolicity and isometries of two generalized hyperbolic metrics, Journal of Natural Science of Heilongjiang University, 24, No. 4 (2007), 498-502. 
